\title{
Fundamental and empirical modelling of co-current spray drying process: a review
}

\begin{abstract}
Spray drying process is frequently being used in food, pharmaceutical, ceramic industries to produce dried powder from bulk liquids. This is a low-temperature and short-time treatment process which involves simultaneous, rapid mass and heat transfer processes which leads to the complexity of the process. Modelling of this process is crucial for design, optimization, control and monitoring purpose. This paper provides review on development of fundamental and empirical models of spray drying process. The essential aspects for categorization of fundamental models is discussed and the most widespread framework for empirical model development is revealed. This review paper will highlight the purposes, advantages and limitations of each model in representing spray drying process.
\end{abstract}

Keyword: Spray drying; Co-current; Modelling; Fundamental model; Empirical model 